Revue critique de fixxion française contemporaine

22 | 2021

Figures du mensonge et de la mauvaise foi dans le roman contemporain

\title{
Mensonge et faux-semblant dans l'autofiction graphique : Faire semblant c'est mentir (2007) par Dominique Goblet
}

\section{Marina Ortrud M. Hertrampf}

\section{(Q) OpenEdition}

\section{Journals}

Édition électronique

URL : https://journals.openedition.org/fixxion/355

DOI : $10.4000 /$ fixxion.355

ISSN : 2295-9106

Éditeur

Ghent University

Référence électronique

Marina Ortrud M. Hertrampf, « Mensonge et faux-semblant dans l'autofiction graphique : Faire semblant c'est mentir (2007) par Dominique Goblet », Revue critique de fixxion française contemporaine [En ligne], 22 | 2021, mis en ligne le 15 juin 2021, consulté le 17 février 2022. URL : http:// journals.openedition.org/fixxion/355; DOI : https://doi.org/10.4000/fixxion.355

Ce document a été généré automatiquement le 17 février 2022.

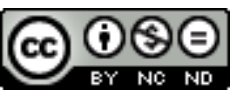

Les contenus de la Revue critique de fixxion française contemporaine sont mis à disposition selon les termes de la licence Creative Commons Attribution - Pas d'Utilisation Commerciale - Pas de Modification 4.0 International. 


\title{
Mensonge et faux-semblant dans l'autofiction graphique : Faire semblant c'est mentir (2007) par Dominique Goblet
}

\author{
Marina Ortrud M. Hertrampf
}

1 Le succès continu des genres littéraires hybrides qui comme l'autofiction ou la docufiction jouent à la fois sur le registre de la fiction et sur celui d'un discours référentiel est aussi un triomphe durable d'une "forme de mensonge spécifiquement littéraire"1. Il n'est donc pas surprenant que cette tendance se soit également imposée au neuvième art. En effet, c'est dans les années 90 que l'autofiction graphique comme sous-genre de la bande dessinée a célébré ses premiers succès en France et en Belgique et reste jusqu'à aujourd'hui un genre extrêmement productif et populaire².

2 À travers l'analyse de l'album Faire semblant c'est mentir $(2007)^{3}$, résultat extraordinaire d'un travail autobiographique réalisé par Dominique Goblet sur douze ans, la présente réflexion s'attachera à montrer comment l'autrice de bandes dessinées belges met esthétiquement en œuvre les thèmes du mensonge, de la mauvaise foi et du fauxsemblant qui dominent la vie de la protagoniste. Le mensonge littéraire ne s'y manifeste pourtant pas seulement au niveau de l'histoire, mais aussi au niveau de la narration même: la bande dessinée raconte - en fragments et de façon non chronologique - deux épisodes autobiographiques. Cependant, la bande dessinée trompe le lecteur dans la mesure où les deux volets narratifs, contrairement aux attentes de celui-ci, ne sont ni directement liés l'un à l'autre ni racontés jusqu'au bout. 


\section{L'autofiction graphique et le mensonge}

\section{L'essor de l'autofiction dans la bande dessinée francophone}

Que les domaines du roman - qui est un genre littéraire - et de la bande dessinée qui est un média artistique - aient convergé récemment dans le concept branché de roman graphique (de l'américain graphic novel), est à la fois normal et surprenant. ${ }^{4}$

3 Ce que Jan Baetens constate ici au sujet du concept du roman graphique en tant que forme particulière de la bande dessinée peut être dit de manière comparable à propos d'une autre forme spécifique du roman graphique qui s'impose de plus en plus depuis quelques décennies sur la scène internationale des arts graphiques et par lequel le genre autobiographique s'incarne dans le média de la bande dessinée : l'autofiction graphique.

4 Le fait même de se représenter ou bien d'introduire des données autobiographiques dans la bande dessinée n'est pas nouveau, mais le traitement artistique des éléments autobiographiques dans la bande dessinée est en revanche innovant. En effet, la démarche autofictionnelle présente une des évolutions les plus remarquables de la bande dessinée : ce qui apparaît au début comme un genre marginal et underground est devenu en quelques décennies un courant majeur de la production du roman graphique actuelle ${ }^{5}$.

5 La tendance qu'a le roman graphique de s'ouvrir à l'autobiographie s'observe, à partir des années 80, d'abord dans le roman graphique américain dont l'exemple le plus célèbre est sans aucun doute celui de Maus d'Art Spiegelman. En réalité, ce n'est que durant les années 90 que le genre prend son essor dans le domaine francophone, surtout avec des auteurs comme Fabrice Neaud et son Journal (1996-2002) ${ }^{6}$, JeanChristophe Menu avec Livret de Phamille (1995) ${ }^{7}$ et ou encore Majane Satrapi avec Persepolis (2000-2003) ${ }^{8}$. En ce qui concerne l'autofiction graphique francophone, il est important de remarquer qu'il ne s'agit pas tout simplement de l'adoption d'une mouvance artistique propre aux États-Unis: "The major influence has not been the model of the American graphic novel but the 'local' model of autofiction - a new way of writing autobiography that blurs the boundaries between the documentary and the fictional"9.

6 Concrètement, il faut distinguer deux raisons qui expliquent ce développement local. L'une d'elle a trait à la volonté de certains auteurs de bande dessinée de dynamiser et revitaliser le médium afin de le libérer de sa "calcification"10. Le développement d'un contre-modèle à la bande dessinée commerciale, qui repose sur la sérialité et le divertissement, ferait de ce médium un art "légitime" car esthétisé : "la bande dessinée a donc vu reconnaître son statut d'art"11. L'émergence de la nouvelle forme de la bande dessinée "se présente ainsi, au début des années 90 , comme un des outils possibles au service d'une avant-garde critique"12.

7 La recherche de nouveaux sujets, de nouveaux formats et de nouveaux styles d'écriture graphique $^{13}$ et littéraire conduit à la création d'une "bande dessinée d'auteur" qui remet régulièrement la vocation fictionnelle du médium en cause. Ce processus s'inscrit en effet dans une vogue considérable des mises en scène du Moi, de l'intimité et du corps dans le champ littéraire ainsi que dans les pratiques de l'art contemporain (de Christian Boltanski à Sophie Calle ou Hervé Guibert) et du cinéma (pensons aux films d'Alain Cavalier ou Romain Goupil). La deuxième explication de l'émergence des 
"autobio-graphismes" (pour retenir un terme forgé par Viviane Alary, Danielle Corrado et Benoît Mitaine ${ }^{14}$ ) francophones est ainsi liée aux nombreux débats autour du concept d'autofiction ${ }^{15}$ et au succès des écritures autofictionnelles.

8 Lorsqu'il s'agit de remettre en question le statut traditionnellement fictif et fictionnel de la bande dessinée et de travailler de manière stylistiquement et esthétiquement innovante, le genre "postmoderne"16 de l'autofiction semble s'imposer : "L'autofiction est un genre expérimental. Dans tous les sens du terme. C'est un laboratoire. Pas la consignation de faits sauce romanesque. Un vrai laboratoire. D'écriture et de vie"17.

\section{L'autofiction graphique et ses relations au mensonge}

Le monde mental

Ment

Monumental

(Jacques Prévert) ${ }^{18}$

9

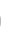

Avec la fictionnalisation du soi et la factualisation de la fiction, l'autofiction se situe volontairement sur un territoire de l'entre-deux: les frontières entre vérités référentielles et mensonges fictionnels (ou bien entre vérités fictionnelles et mensonges référentiels) sont toujours floues et disparaissent souvent entièrement ${ }^{19}$. Sans même se référer aux dimensions morales ou éthiques auxquelles invite cette ambiguïé, l'autofiction est une forme narrative qui se veut insaisissable et dont la mauvaise foi est systématique et inhérente au genre :

Dans le cas du texte d'autofiction, le "mensonge" provient de l'utilisation savante d'éléments fictionnels dans le cadre d'un projet autobiographique, jeu élaboré reposant sur des procédés romanesques qui sont mis en œuvre pour chercher à atteindre une plus grande fidélité au réel. ${ }^{20}$

Or, l'autofiction graphique multiplie ce statut paradoxal de l'autofiction. La bande dessinée a la même souveraine liberté que la littérature pour fictionnaliser le "je" auteur-narrateur-protagoniste et pour "reconstruire un passé qu'elle ne prétend pas avoir enregistré. Le dessin comme l'écriture ne sont pas des traces, mais des signes" 21 .

11 L'identité entre les trois instances de l'auteur, du narrateur et du protagoniste ${ }^{22}$ est cependant bien plus douteuse que dans l'autofiction littéraire, car en se dessinant en tant que personnage, le narrateur-auteur d'une bande dessinée autobiographique réintroduit la dimension du "il" ${ }^{23}$. L'identité entre auteur et personnage est donc très relative. Bien évidemment, l'un représente l'autre, "mais la monstration sur laquelle repose la bande dessinée le réifie en tant qu'acteur' doué d'une identité propre, elle le fait 'jouer' et le met en scène"24. Il y a donc nécessairement une scission entre le moiauteur et le moi-personnage dessiné.

12 Jean-Christophe Menu - rédacteur en chef du célèbre éditeur de bandes dessinées L'Association, l'un des principaux promoteurs du mouvement autobiographique dans les romans graphiques francophones et auteur de l'album autofictionnel Livret de Phamille - énonce très bien cette idée :

Si je m'autoreprésente, ce n'est pas une image ayant un rapport réel avec "moi" qui sort de ma plume, c'est le plus souvent un symbole, un condensé hiératique (même au niveau temporel : c'est une espèce de synthèse des différents âges que j'ai eus). [...] D'ordinaire, ce qui survient est l'autoarchétype, qui permet d'avancer le récit sans se poser la question de la représentation..$^{25}$

Revue critique de fixxion française contemporaine, 22 | 2021 
L'autoportrait graphique que crée un auteur ne représente donc le plus souvent qu'une (re-)construction graphique stylisée, fragmentée, parfois même arbitraire, du moi, qui est étroitement liée au processus de création artistique et qui reste donc toujours incomplète et dynamiquement modifiable. Fabrice Neaud spécifie en ces termes le processus de création procédural du moi-personnage dessiné :

Le "je" autobiographique n'est pas un "déjà là". Il n'est pas une figure finie délimitée par ses patterns graphiques pas plus que par son positionnement moral: il est un être en devenir. [...] Notre époque encore empreinte d'essentialisme, doublée de la misère propre de la bande dessinée à se voir comme un médium s'adressant toujours à des neuneus, a du mal à concevoir qu'un être, que "soi", puisse être non pas une essence finie mais un processus, soit une construction en devenir. ${ }^{26}$

Il s'agit alors très souvent plus d'une approche "symbolique", comprise comme une tentative auto-définitoire d'introspection, que d'une approche référentielle qui tente d'établir une ressemblance physique photo-réaliste. Autoreprésentation plutôt qu'autoportrait, le moi-personnage comme entité graphique s'écarte délibérément du mimétisme et de la vérisimilitude et, en ce sens, se rapproche du mensonge littéraire.

\section{Faire semblant c'est mentir : une analyse des dimensions mensongères}

\section{Dominique Goblet : à la recherche des vérités de sa vie}

Dominique Goblet (*1967), fille d'un père wallon et d'une mère flamande, est illustratrice, autrice de bandes dessinées et plasticienne belge. Après ses premiers romans graphiques - Portraits crachés $(1997)^{27}$ et Souvenir d'une journée parfaite $(2002)^{28}$, Faire semblant c'est mentir (2007) est sans aucun doute le chef-d'œuvre qui lui a permis de se faire connaître sur la scène internationale de la bande dessinée : jusqu'à présent, l'album a été traduit en allemand, en anglais et en russe. Elle est aussi l'autrice des albums Chronographie (avec sa fille Nikita Fossoul ; 2010), Les hommes-Loups (2010), Plus si entente (avec Kai Pfeiffer ; 2014) et L'amour dominical (avec Dominique Théate ; 2019) ${ }^{29}$. En 2020, Dominique Goblet reçut, outre le Prix de la Fédération Wallonie-Bruxelles en bande dessinée, le Grand Prix Töpffer pour l'ensemble de son œuvre graphique.

Avec le soutien de Jean-Christophe Menu, Dominique Goblet s'est engagée dans un travail de mémoire troublant et sans concession, aussi bien au sujet d'elle-même qu'au sujet de ses proches. Dans les remerciements, Dominique Goblet souligne que sans JeanChristophe Menu, "ce livre n'existerait pas", et précise qu'il

a joué un rôle dépassant largement celui d'un éditeur et d'un ami exceptionnel et qui, par ses réflexions et ses sensibles questions m'a poussée, quand il le fallait, à remettre mon travail en question et m'a permis d'aller au bout de ce que j'avais à faire, vraiment tout au bout. (FSM s.p.)

Lancé en 1995, Faire semblant c'est mentir est le résultat singulier d'une recherche autobiographique à la fois difficile et intense de la vérité, et que Dominique Goblet a réalisée sur douze ans. Notamment en raison de son processus de création inhabituellement long, l'album reflète également le développement artistique de l'autrice pendant cette période de sa vie, et contribue à faire de Faire semblant c'est mentir une véritable autobiographie d'auteur au niveau formel et stylistique ${ }^{30}$. Son 
“mentor" Jean-Christophe Menu décrit lui-même l'ouvrage comme tel dans son avantpropos:

Les événements ont fait que cette Autobiographie a été en mise en suspens, plusieurs fois. Il y a eu d'autres livres, des expositions, des voyages, l'Autobiographie est revenue, repartie, revenue. La manière avait changé, le crayon gris fixait Bruxelles et Charleroi avec stabilité, mais c'était la même histoire que les planches de 1995, qui avaient continué à jaunir de leur côté. Réintégrer leur sépia et leur style désormais ancien à un présent nouveau était une façon pour Dom de défier le temps, vraie matière de ce livre, achevé douze ans après son commencement. (FSM s.p.)

17 Le roman graphique retrace alors certains moments clés de l'enfance et de la vie d'adulte de Dominique Goblet qui sont fortement liés au faux-semblant et au mensonge : d'un côté ce sont des expériences douloureuses de l'autrice-protagoniste comme jeune fille, liées notamment à l'alcoolisme du père et aux violences faites à la mère; de l'autre, ce sont des expériences perturbantes avec son amant Guy Marc Hinant, qui est uniquement désigné dans l'album par l'abréviation GM et qui ne parvient pas à oublier sa relation antérieure.

Faire semblant c'est mentir est par conséquent la tentative artistique d'une autrice qui cherche à retracer les vérités de sa vie, une vie toujours dominée par la mauvaise foi et le mensonge : sa mère, qui est mentalement instable et malheureuse dans son mariage, est capable de cacher les petits problèmes de la vie quotidienne, mais en même temps cache les abus de sa fille; son père, un menteur notoire, prétend avoir toujours été un père aimant et fidèle et nie son alcoolisme ; son compagnon fait semblant de l'aimer bien qu'il soit toujours très attaché à son ex-petite amie. Finalement, les fauxsemblants finissent par masquer la réalité et les personnages finissent par mentir à tour de rôle, à eux-mêmes comme à leur entourage.

\section{La multiplication narrative et discursive du mensonge}

Dans l'autofiction graphique, il n'y a pas que le dessin et l'écriture qui sont liés de façon indissoluble, mais également la vie réelle, la mémoire et l'imagination. Ainsi, le métissage de la fiction et de la réalité, du mensonge et de la vérité, produit une fictionnalisation de soi à travers laquelle l'auteur-dessinateur-narrateur crée une nouvelle identité qui parfois démasque des impostures identitaires et révèle des vérités dissimulées. Telle est la démarche adoptée par Faire semblant c'est mentir, où le lecteur est confronté aux menteurs et aux mensonges au niveau de l'histoire.

L'oscillation entre vérité et mensonge, véracité et mystification se répercute pourtant aussi au niveau du discours et de l'écriture graphique. Dans ce contexte, le titre suggère une certaine ambiguïté.

21 Il fait d'abord référence aux expériences douloureuses de l'autrice. L'affirmation selon laquelle "faire semblant c'est mentir" représente finalement la conclusion impitoyable de l'autrice après son auto-réflexion critique sur ses relations interpersonnelles. Le fait qu'il s'agisse d'une conclusion à laquelle l'autrice n'est venue que rétrospectivement est illustrée de manière très claire par la scène présentée dans l'“Introduction". Sur quatre planches à six vignettes chacune, Dominique Goblet raconte avec une douceur amoureuse comment sa mère l'a réconfortée avec un petit mensonge : la petite est tombée et a déchiré ses collants. La mère les lui enlève, fait semblant de pouvoir faire 
de la magie et les met sur l'enfant dans le mauvais sens, de sorte que la petite Dominique croit réellement que la mère a réparé les trous par magie.

Cette scène inoffensive montre cependant que même les petites manœuvres de tromperie innocentes ne sont rien d'autre que des mensonges. Ce qui est présenté ici de manière positive, depuis le point de vue de l'enfant, s'avère paradigmatique, aux yeux de la Dominique adulte, d'une vie dominée par le mensonge.

Mais le titre renvoie aussi à l'œuvre elle-même sous la forme d'une mise en abyme et là encore se manifeste une certaine ambiguiité. D'un côté, le titre fait allusion au mélange de fiction et de réalité, et donc au statut générique de l'autofiction en tant que genre intimement lié au mensonge. Se dessiner n'est jamais plus que reconstruire un "autoarchétype" fictionnel de soi-même, ce n'est donc rien d'autre que faire semblant. En effet, il s'agit là d'un mensonge par omission et distorsion : "l'auteur sélectionne et ordonne, omet ce qui le gêne, et ce faisant leurre son lecteur puisque le personnage ainsi révélé reste personnage littéraire, une illusion, un mirage" ${ }^{31}$. Ceci est également explicitement mentionné par Guy Marc Hinant, avec qui Dominique Goblet a co-écrit les textes des chapitres 2 et 4 , dans son épilogue intitulé "Initiales, outils, simulacres" :

Le passé est fiction, re-mémorisation, ré-interprétation, fixation momentanée (sur base d'une réalité admise), projection, hypothèse, opacité. Notre passé charrie, comme vrai, des souvenirs où se mêle, le plus souvent, ce qui nous a été rapporté bien des fois (les histoires récurrentes des parents). (FSM s.p.)

Enfin, Guy Marc Hinant prend position sur ses relations avec son double fictif dans la bande dessinée :

Son aspect trouble et mensonger n'échappe à ma possible opprobre que par cette mise à distance qu'est le travail d'écriture - c'est bien un personnage (dont les composantes de culpabilité, effroi, douleur, etc.) que je tente de faire vivre dans la structure du livre. Ainsi devenons-nous intelligibles et sans regret. Tout peut s'aborder sans peur, ni remords. Pourquoi ? Parce qu'il ne s'agit pas de la vie ellemême mais de l'Art (omnisciente, inattaquable puissance de l'art). C'est pourquoi GM n'est pas Guy Marc et que la Dom du récit n'est pas Dominique Goblet - ce sont, en réalité, des avatars contrôlés par des personnes vivantes portant des noms similaires. (FSM s.p.)

Revenons à nos réflexions sur l'ambiguïté du titre : la conclusion selon laquelle "faire semblant c'est mentir" implique une situation similaire pour l'autrice-narratrice ; mais elle conduirait alors de manière paradoxale au constat d'une menteuse faisant semblant de raconter sa vie. Cependant, cela signifierait également que l'autricenarratrice ne peut que mentir en disant la vérité ou dire la vérité en mentant. La valeur aléthique d'une telle proposition est évidemment indécidable. Sachant qu'un menteur ne dit jamais la vérité, le titre serait-il donc forcément un mensonge ? Avec un contrat de lecture aussi contradictoire, il reste impossible pour le lecteur de faire la part entre mensonge et fiction. Comment savoir à quel point la vie de Dominique Goblet ressemble à ce qu'elle en dit et se montre dans sa propre autobiographie graphique ? La vérité de l'album reste donc en jeu.

De l'autre côté, le titre peut également être compris comme méta-référentiel, car Dominique Goblet suggère ici une incapacité narrative et graphique, qui n'est bien sûr que simulée et qui est consciemment utilisée comme dispositif stylistique, reflétant le mensonge et la tromperie au niveau de la forme.

Dans des chapitres apparemment sans lien, Faire semblant c'est mentir raconte des relations familiales qui dysfonctionnent, et cela d'une manière très fragmentaire, non 
linéaire et non chronologique. À première vue, l'autrice-narratrice ne semble pas être capable de raconter de manière cohérente, de sorte qu'au départ, ni la connexion chronologique, ni la connexion logique des épisodes narratifs individuels ne sont révélées. Mais ce n'est qu'une tromperie superficielle. Petit à petit, on comprend quelle est la logique qui tient ensemble les épisodes narratifs au sujet du père et de la mère, de l'éducation, du nouveau partenaire et de l'expérience de la maternité ; à savoir ce que le personnage de Dominique Goblet elle-même n'a pas vécu en tant qu'enfant ou en tant qu'adulte : stabilité émotionnelle et affection, confiance et honnêteté. En outre, la protagoniste s'interroge sur sa capacité à offrir tout ceci à sa propre fille Nikita.

Une procédure similaire peut également être observée sur le plan graphique : l'aspect brouillon, la simplicité d'un trait presque enfantin, le manque de perspective et les changements de style ${ }^{32}$ laissent deviner au lecteur une incapacité artistique qui, bien évidemment, n'est que simulée. Le style simple, parfois grossier et maladroit, des dessins prétend provenir de la main du moi-personnage enfantin. Par conséquent, il fonctionne comme une sorte de voyage dans le temps, comme une mise en scène artistique de la psyché du moi-personnage enfantin, et, finalement, réverbère le travail de la mémoire profonde du moi-auteur.

En regardant la représentation graphique des personnages, il devient clair que Dominique Goblet s'appuie délibérément sur des formes de représentation non réalistes. Sa représentation d'elle-même, comme celle de ses proches, est très abstraite et ressemble parfois à des caricatures, voire à des figures grotesquement déformées. Le fait que l'autrice se peigne sans prêter attention aux détails et de manière plutôt caricaturale évoque une forme de dureté à l'égard d'elle-même. Dans le même temps cependant, cela semble aussi être le résultat d'un manque de reconnaissance de la part de son père qui l'appelle Nikske - le diminutif habituel de Dominique en Bruxellois (comme l'explique l'auteur); et le fait que cela signifie "petit rien" montre sans ambages que son éducation n'a pas permis de lui donner confiance en elle.

Ces dessins déformés et grotesques trouvent de surcroît un écho dans l'utilisation extraordinaire du lettrage. À l'aide d'un travail typographique sur la taille, les polices, l'épaisseur, l'alternance de lettres précises ou maladroites, Dominique Goblet donne aux signes arbitraires de la langue des qualités déictiques et iconiques. Le lettrage y fonctionne comme partie intégrante du protagoniste, au même titre que les membres de son corps; tous deux se tordent de façon inquiétante ou prennent des formes monstrueuses. Tandis que les personnages se caractérisent par leurs énoncés linguistiques, l'autrice les caractérise extérieurement via le lettrage et transpose sa focalisation interne, c'est-à-dire sa perception des personnes et de ses émotions, à l'échelle des lettres. Cela se voit, par exemple, dans les lettres agressives et griffonnées par lesquelles Cécile, la compagne du père de Dominique, exprime sa colère envers Nikita.

En même temps, cet exemple montre aussi très bien à quel point la focalisation interne est ici rendue visible: au lieu d'une représentation réaliste, Dominique Goblet transfigure le visage de Cécile en une sorte de masque mortuaire. Cela met en évidence non seulement la relation froide et sans émotion entre Dominique et Cécile, mais aussi l'hypocrisie de Cécile, qui cache son vrai visage derrière un masque rigide. Cette feintise mensongère s'exprime, entre autres, dans le fait qu'elle aborde Dominique et sa fille avec une politesse simulée, mais en usant d'un ton plein de dédain et d'agressivité à leur encontre. 
31 L'élément déclencheur de l'attaque de Cécile contre Nikita est d'ailleurs à nouveau le mensonge. Bien qu'elle n'agisse pas elle-même différemment, elle condamne l'interprétation inconstante de Nikita du dessin qu'elle a peint pour Cécile. Cette scène souligne comment le style du dessin témoigne de la relation émotionnelle entre les personnes telle qu'elle est perçue par l'autrice. Alors que Cécile est représentée en conservant des proportions normales dans la première vignette de la planche, dans les vignettes suivantes, elle se transforme en bonhomme menaçant, à tête de mort, démesurément grand et semblable à une araignée. Nikita, elle, ou plutôt son dessin n'apparaît que comme une minuscule figure tout en bas de la vignette. En contraste avec cette représentation grotesque de Cécile, la vignette dans laquelle Dominique est vue avec sa fille paraît presque réaliste et met en avant une relation mère-fille beaucoup plus facile, voire harmonieuse.

Cette scène est d'autant plus intéressante que Nikita y devient un double de Dominique $^{33}$ : Nikita doit supporter le même comportement agressif que sa mère a connu depuis sa propre enfance. De plus, la scène peut aussi être lue comme une mise en abyme, car après tout, il s'agit de vérité et de mensonge quand on dessine de vraies personnes. Ce n'est donc pas un hasard si le titre de l'album est le thème central de cette scène dans laquelle le dessin autobiographique est dominant.

C'est en effet de mensonges et d'hypocrisie que Dominique accuse son père. Mais le père ne peut accepter ces allégations et accuse sa fille de mensonge (et d'ingratitude). L'autrice en donne un exemple particulièrement impressionnant lorsqu'elle illustre un discours sur la défensive de son père sur une page entière, délaissant tout cadre réaliste. Dominique Goblet transcrit alors l'auto-glorification de son père en tant que (faux) saint, en tant que père aimant qui, après la séparation de sa mère, prend soin de sa fille avec dévouement, en dépeignant le père à la manière byzantine de Notre-Dame intronisée et en reproduisant son discours dans des lettres gothiques évoquant les miniatures des manuscrits bibliques médiévaux. Si le regard sérieux du père correspond à la tradition iconographique des icônes, le regard effrayé de l'enfant est aussi étonnant que le fait qu'il ne soit pas - contrairement à la tradition - entouré d'un halo. Cela semble combiner l'auto-perception du moi-personnage (Dominique ne se voit pas comme une sainte) et la perception externe du père qui accuse sa fille d'être une menteuse. Il est également intéressant de noter que l'auto-glorification mensongère du père est liée à une critique de l'hypocrisie de l'Église catholique, qui joue un rôle majeur en Belgique et pourrait promouvoir une forme d'hypocrisie dans la sphère privée de la famille. Malgré tous les problèmes entre père et fille, l'ambiguïté de leurs sentiments, qui est certainement aussi celle de l'amour et de l'affection, transparaît de la sorte.

Enfin, on peut voir une dernière forme du faux-semblant graphique dans le fil narratif sur GM. Tout en étant avec Dominique, ce dernier pense constamment à son ex-petite amie, mais s'en cache en omettant d'en parler. Ses mensonges ne sont pas formulés verbalement, mais ils sont visuellement mis en scène de telle sorte que l'imaginaire, la présence mentale et émotionnelle de l'ex-petite amie de GM se matérialise sous la forme d'un fantôme, une ombre presque toujours présente. Ce que la protagoniste ne sait ni ne soupçonne devient visible au lecteur, car la focalisation interne sur GM est représentée graphiquement. Ainsi, le lecteur sait que GM la trompe avant que la protagoniste ne le découvre. Le dispositif graphique dévoile donc la vérité, en l'occurrence le mensonge : faire semblant c'est mentir. 


\section{Conclusion : la vérité au-dessus du mensonge}

Faire semblant c'est mentir est une autofiction graphique singulière à de multiples égards qui "ne respire comme aucun autre livre" (FSM s.p.), conclut Menu dans son avantpropos. En effet, c'est cette forte émotivité exprimée par Dominique Goblet dans un style graphique idiosyncratique et expressif, sans nombrilisme ni sentimentalité, qui rend l'autofiction graphique "vraie". Il en va de même de la réduction du texte verbal au profit des différents langages picturaux, liant ainsi le refoulé et le sublimé à travers le dessin, dans le silence des mots, mais cela de manière beaucoup plus directe (et donc plus vraie). Après tout, ce sont précisément les pauses, les anachronies, les omissions et les allusions ${ }^{34}$ qui exhibent la vérité sous le mensonge.

En conclusion, il faut donc noter que, contrairement à Persépolis de Majane Satrapi, Faire semblant c'est mentir ne cherche pas à relier l'intime aux grands contextes sociopolitiques de manière documentaire. Au lieu de cela, Dominique Goblet choisit une voie littéraire profondément artistique et esthétique : avec une cohérence impressionnante, elle plonge dans les profondeurs de sa vie afin d'en bannir les pensées et les sentiments censément authentiques. À cet égard, l'hypothèse de l'autobiographie se révèle être une mauvaise piste à suivre. L'autrice met plutôt en relief dans son récit rétrospectif les conséquences destructrices des mensonges et des impostures identitaires pour la psyché de l'individu en général.

Malgré toute sa dureté, et malgré toutes les blessures et les déceptions qu'elle dépeint, la bande dessinée parfois très sombre et mélancolique ne se termine pas de manière pessimiste. Bien au contraire: le travail de mémoire douloureux et la remise en question incessante de soi conduisent en dernière instance à un sentiment de libération et de soulagement, symbolisé par de larges planches de couleur beige clair et ocre qui rappellent vaguement la peinture abstraite des champs de couleurs de Mark Rothko ${ }^{35}$. En fin de compte, les âmes du moi-personnage et de GM se détournent de tous les mensonges et fantômes. Elles sont libérées comme des hirondelles en vol (dans le ciel que regarde GM depuis son nouvel appartement après s'être séparé de la protagoniste) et semblent pleines d'optimisme en regard d'un avenir commun. Dans ce contexte, les fragments de dialogue d'une conversation téléphonique entre GM et Dominique Goblet sont particulièrement frappants. On note d'une part la déclaration d'intention “... POUR NE PAS TE MENTiR ..." (FSM s.p.) et de l'autre le commentaire qui évoque un oiseau blessé par un chat que GM tient dans la main mais qui se détache du contexte immédiat de l'action par son intégration dans une page de couleur pastel. C'est pourquoi ce commentaire peut aussi se référer à $\mathrm{GM}$, au mensonge et au fantôme de cette relation amoureuse avec Dominique Goblet dont le charme a fini par se rompre :

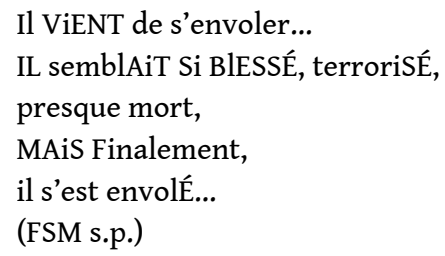

Ainsi la bande dessinée se ferme littéralement (et avec un point) dans le "maintenant" et donc dans la vraie réalité ? 


\section{NOTES DE FIN}

1. Jochen Mecke, "Esthétique du mensonge", Cahiers d'Études Germaniques, n 68, 2015, p. 83, disponible sur <http://journals.openedition.org/ceg/1436> (consulté le 15 janvier 2021).

2. Laurent Gerbier, “'Se donner un genre'. Grandeur et décadence de l'autobiographie dessinée”, in Viviane Alary, Danielle Corrado, Benoit Mitaine (dirs), Autobio-graphismes : Bande dessinée et représentation de soi, Chêne-Bourg, Georg, 2015, p. 29.

3. Dominique Goblet, Faire semblant c'est mentir, Paris, L'Association, 2007 ; dorénavant FSM.

4. Jan Baetens, "Le roman graphique”, in Éric Maigret, Matteo Steffanelli (dirs), La bande dessinée : une médiaculture, Paris, Armand Colin, 2012, p. 200.

5. Voir Jan Baetens, “Autobiographies et bandes dessinées", Belphégor, n 4, vol. 1, 2004, disponible sur <http://dalspace.library.dal.ca//handle/10222/47689> (consulté le 15 janvier 2021) ; Thierry Groensteen, “Autobiographie", Neuvième art 2.0, 2014, disponible sur <http:// neuviemeart.citebd.org/spip.php?article813> (consulté le 15 janvier 2021); Catherine Mao, (2013), "L'artiste de bande dessinée et son miroir : l'autoportrait détourné", Comicalités, 2013, disponible sur <http://comicalites.revues.org/1702> (consulté le 15 janvier 2021) ; Viviane Alary, Danielle Corrado, Benoit Mitaine, Autobio-graphismes: Bande dessinée et représentation de soi, Chêne-Bourg, Georg, 2015.

6. Fabrice Neaud, Journal : Février 1992 - septembre 1993, Angoulême, ego comme x, 1996 ; Journal : Septembre 1993 - décembre 1993, Angoulême, ego comme x, 1998 ; Journal : Décembre 1993 - août 1995, Angoulême, ego comme x, 1999 ; Journal : Les Riches heures, Angoulême, ego comme x, 2002.

7. Jean-Christophe Menu, Livret de Phamille, Paris, L'Association, 1995.

8. Marjane Satrapi, Persepolis, tome 1, Paris, L'Association, 2000 ; Persepolis, tome 2, Paris, L'Association, 2001; Persepolis, tome 3, Paris, L'Association, 2002 ; Persepolis, tome 4, Paris, L'Association, 2003.

9. Jan Baetens, "Dominique Goblet. The List Principle and the Meaning of Form", in Michael A. Chaney (dir.), Graphic Subjects. Critical Essays on Autobiography and Graphic Novels, Madison (Wisconsin), The Univeristy of Winconsin Press, 2011, p. 76.

10. Jean-Christophe Menu, Fabrice Neaud, “Autopsie de l'autobiographie”, L'Éprouvette, $\mathrm{n}^{\circ} 3$, Paris, L'Association, 2007, p. 454.

11. Philippe Lejeune, “Avant-propos”, Viviane Alary, Danielle Corrado, Benoit Mitaine (dirs), Autobio-graphismes, op. cit., p. 12.

12. Laurent Gerbier, art. cit., p. 29.

13. Pour une introduction aux formes narratives traditionnelles et innovantes en bande dessinée voir Thierry Groensteen, Comics and narration, Jackson, University Press of Mississippi, 2013.

14. Viviane Alary, Danielle Corrado, Benoit Mitaine, "Introduction : Et moi, émoi !", in Viviane Alary, Danielle Corrado, Benoit Mitaine (dirs), Autobio-graphismes, op. cit., p. 20.

15. Pour un survol des débats théoriques du concept voir notamment Garcia Mar, "L'étiquette générique 'autofiction' : us et coutumes", Çédille: Revista de Estudios Franceses, n 5, 2009, disponible sur <https://www.researchgate.net/publication/ 26598747_L\%27etiquette_generique_autofiction_us_et_coutumes> (consulté le 15 janvier 2021).

16. Doubrovsky souligne l'actualité du genre en disant : "L'autofiction est la forme postmoderne de l'autobiographie." Serge Doubrovsky, "Les points sur les 'i”', in Jean-Louis Jeannelle, Catherine Viollet, Isabelle Grell (dirs), Genèse et autofiction, Louvain-la-Neuve, Academia-Bruylant, 2007, p. 64-65.

17. Chloé Delaume, La règle du Je, Paris, Presses Universitaires de France, 2010, p. 20.

18. Il s'agit des trois derniers vers du poème "Il ne faut pas" de Jacques Prévert, Paroles, Paris, Les Éditions du point du jour, 1946. 
19. Parmi le très grand nombre de publications sur l'autofiction, seuls deux articles sont mentionnés ici : Laouyen, Mounir, "L’autofiction : une réception problématique", in René Audet, Alexandre Gefen (dirs), Frontières de la fiction, Pessac, Presses Universitaires de Bordeaux, 2002, p. 339-356, disponible sur <http://books.openedition.org/pub/5765> (consulté le 15 janvier 2021) ; Philippe Vilain, L'Autofiction, exception théorique; Marc Dambre, Richard J. Golsan (dirs), L'exception et la France contemporaine: Histoire, imaginaire et littérature, Paris, Presses Sorbonne Nouvelle, 2010, p. 161-168, disponible sur <http://books.openedition.org/psn/339> (consulté le 15 janvier 2021).

20. Sylvie Lannegrand, "La poursuite d'une représentation impossible: écriture autobiographique, mensonge et vérité chez Robbe-Grillet", in Kate Averis, Matthew Moran (dirs), Le Mensonge: Multidisciplinary Perspectives in French Studies, Cambridge, Cambridge Scholars Publishing, 2010, p. 65.

21. Philippe Lejeune, art. cit., p. 12.

22. Aspects constitutifs de l'autobiographie selon Philippe Lejeune, Le pacte autobiographique, Paris, Seuil, 1975, <Poétique>, p. 4.

23. Pour la multiplication des niveaux de l'ego dans les autofictions graphiques voir Philippe Maupeu, "Présentation", in Philippe Maupeu (dir.), Territoires autobiographiques : récits-enimages de soi, Toulouse, Presses Universitaires du Midi, 2018, p. 9-19, disponible sur <https:// doi.org/10.4000/litteratures.1806> (consulté le 15 janvier 2021).

24. Thierry Groensteen, "Problèmes de l'autoreprésentation", in Viviane Alary, Danielle Corrado, Benoit Mitaine (dirs), Autobio-graphismes, op. cit., p. 54.

25. Jean-Christophe Menu, Christian Rosset, Correspondance, Paris, L'Association, 2009, p. 10.

26. Jean-Christophe Menu, Fabrice Neaud, art. cit., p. 472.

27. Dominique Goblet, Portraits crachés, Bruxelles, Fréon, 1997. Pour une analyse de Portraits crachés voir Jan Baetens, "Dominique Goblet. The List Principle and the Meaning of Form", art. cit.

28. Dominique Goblet, Souvenir d'une journée parfait, Bruxelles, Fréon, 2002.

29. Dominique Goblet, Nikita Fossoul, Chronographie, Paris, L'Association, 2010; Dominique Goblet, Les hommes-loups, Bruxelles, Frémok, 2010 ; Dominique Goblet, Kai Pfeiffer, Plus si entente, Bruxelles, Frémok, 2014 ; Dominique Goblet, Dominique Théate, L'amour dominical, Bruxelles, Frémok, 2019.

30. Depuis le début de sa production graphique, Dominique Goblet a été l'une des autrices de bandes dessinées autobiographiques avant-gardistes qui rompent avec la sérialité ainsi qu'avec les autres structures narratives traditionnelles pour tendre davantage vers l'hétérogénéité stylistique: "Albums such as those by Fabrice Neaud (especially his Journal 4) or Dominique Goblet, to give but two examples, show that breaks in style are now accepted, and have indeed become an integral part of the artist's array of rhetorical resources. This achievement by modern comics facilitates, it would seem, the involvement of the monstrator." (Thierry Groensteen, Comics and narration, Jackson, University Press of Mississippi, 2013, p. 94). Cependant, Dominique Goblet diffère des autres auteurs de romans graphiques autobiographiques non seulement en raison de leur perspective féminine: "Despite the presence of themes such as domestic violence and rape, it is clear that she emphasizes to the same degree the technical aspects of her artwork, clearly indepted to modernism and the avant-garde, in addition to the thematic and ideological aspects of her storyworld." (Jan Baetens, "Dominique Goblet. The List Principle and the Meaning of Form", art. cit., p. 78). Pour une analyse du style graphique de Dominique Goblet, voir Gert Meesters, "Les significations du style graphique: 'Mon fiston' d'olivier Schrauwen et 'Faire semblant c'est mentir' de Dominique Goblet”, Textyles : Revue des Lettres Belges de Langue Française, $\mathrm{n}^{\circ} 36-37,2010$, p. 215-233. 
31. Sylvie Lannegrand, "La poursuite d'une représentation impossible: écriture autobiographique, mensonge et vérité chez Robbe-Grillet”, in Kate Averis, Matthew Moran (dirs), op. cit., p. 65.

32. Complexes, flous et négligés, les dessins sont extrêmement hétérogènes. Le crayon et le stylo à bille leur donnent volontiers l'apparence de brouillons, mais parfois ces croquis se mélangent à la peinture à l'huile. Souvent, des traces de révisions et de corrections sont même visibles. Des dessins de type collage, rappelant les dessins d'enfants, côtoient des compositions d'une densité artistique inouie. La colorisation est tout aussi hétérogène, passant de dessins en noir et blanc (presque) monochromes à des jeux de couleurs en nuances de brun et marron clair.

33. Cela montre également le fait que l'enfant Dominique et Nikita sont dessinées de manière très similaire. La représentation mignonne avec la grosse tête et les grands yeux rappelle le style Manga.

34. En effet, il y a plusieurs planches quasi abstraites qui se réfèrent à la peinture non-figurative : "ces images [...] offrent une pause dans le récit, un moment de réflexion. Les grandes cases dans une bande dessinée ont la caractéristique de ralentir le rythme de la narration." (Gert Meesters, art. cit., p. 222-223).

35. "D'après l'autrice elle-même, ces couleurs réfèrent au ciel de Bruxelles, qui peut sembler somber ou plein d'espoir selon les circonstances." (ibid., p. 223).

\section{RÉSUMÉS}

Le succès continu des genres littéraires hybrides qui comme l'autofiction ou la docufiction jouent à la fois sur le registre de la fiction et sur celui d'un discours référentiel se manifeste également en neuvième art. En effet, c'est dans les années 1990 que l'autofiction graphique comme sousgenre de la bande dessinée a célébré ses premiers succès dans le monde francophone et reste jusqu'à aujourd'hui un genre extrêmement productif et populaire.

À travers une analyse de l'album Faire semblant c'est mentir (2007), résultat extraordinaire d'un travail autobiographique réalisé par Dominique Goblet sur douze ans, le présent article s'attache à montrer comment l'auteure de bandes dessinées belge met en œuvre esthétiquement les thèmes du mensonge et du faux-semblant. En effet, il s'avère que la vie de la protagoniste était et est toujours dominée par la mauvaise foi et le mensonge. Le mensonge littéraire ne s'y réfère pourtant pas seulement au niveau de l'histoire, mais aussi au niveau de la narration même : la bande dessinée raconte - en fragments et de façon non chronologique - deux épisodes autobiographiques. Cependant, la bande dessinée trompe le lecteur dans la mesure où les deux volets narratifs, contrairement à l'attente du lecteur, ne sont ni directement liés l'un à l'autre ni racontés jusqu'au bout.

\section{INDEX}

Mots-clés : neuvième art, autofiction graphique, faux-semblant, mensonge

\section{AUTEURS}

\section{MARINA ORTRUD M. HERTRAMPF}

Université de Passau 